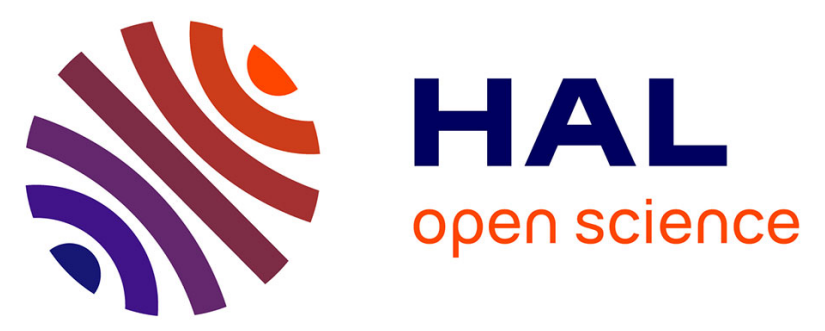

\title{
Republication de: Facteurs organisationnels associés à la réalisation d'une césarienne dans une population à bas risque
}

\author{
C. Duvillier, A. Rousseau, C. Bouyer, F. Goffinet, P. Rozenberg
}

\section{- To cite this version:}

C. Duvillier, A. Rousseau, C. Bouyer, F. Goffinet, P. Rozenberg. Republication de: Facteurs organisationnels associés à la réalisation d'une césarienne dans une population à bas risque. La Revue Sage-Femme, 2019, 18, pp.14 - 23. 10.1016/j.sagf.2019.01.003 . hal-03486312

\section{HAL Id: hal-03486312 https://hal.science/hal-03486312}

Submitted on 20 Dec 2021

HAL is a multi-disciplinary open access archive for the deposit and dissemination of scientific research documents, whether they are published or not. The documents may come from teaching and research institutions in France or abroad, or from public or private research centers.
L'archive ouverte pluridisciplinaire HAL, est destinée au dépôt et à la diffusion de documents scientifiques de niveau recherche, publiés ou non, émanant des établissements d'enseignement et de recherche français ou étrangers, des laboratoires publics ou privés.

\section{(ㄷ)(1) $\$$}

Distributed under a Creative Commons Attribution - NonCommercial| 4.0 International 
Mise au point

\title{
Facteurs organisationnels associés à la réalisation d'une césarienne dans une population à bas risque
}

\section{Impact of organizational factors on the cesarean delivery occurrence in a low-risk population}

\author{
C. Duvillier ${ }^{\mathrm{a}}$, A. Rousseau ${ }^{\mathrm{a}}, \mathrm{C}$. Bouyer ${ }^{\mathrm{b}}, \mathrm{F}$. Goffinet ${ }^{\mathrm{c}}$, P. Rozenberg $^{\mathrm{a}, \mathrm{b}, \mathrm{d}, *}$ \\ ${ }^{a}$ Unité de recherche EA 7285, université de Versailles Saint-Quentin-en-Yvelines, 2, avenue de la source de la Bièvre, 78180 Montigny-le-Bretonneux, France \\ ${ }^{\mathrm{b}}$ Réseau maternité en yvelines et périnatalité active (MYPA), Pavillon Courtois, 20, rue Armagis, 78100 Saint-Germain-en-Laye, France \\ ${ }^{\mathrm{c}}$ Maternité Port-Royal, Assistance publique-Hôpitaux de Paris, 123, boulevard de Port-Royal, 75014 Paris, France \\ ${ }^{\mathrm{d}}$ Service d'obstétrique et gynécologie, centre hospitalier Poissy/Saint-germain, 10, rue du Champ-Gaillard, 78300 Poissy, France
}

\section{N F O A R T I C L E}

\section{Historique de l'article :}

Reçu le 18 mai 2018

\section{Mots clés :}

Césarienne

Facteurs organisationnels

Population à bas risque

Classification de Robson

\section{Keywords:}

Cesarean delivery

Organizational determinants

Low-risk population

Robson classification

\begin{abstract}
R É S U M É
Objectif. - Il existe des différences considérables du taux de césariennes entre les maternités d'une même région. Le taux de césarienne a augmenté ces dernières années dans la population à bas risque. L'objectif de cette étude était d'étudier l'impact des facteurs organisationnels sur le taux de césarienne dans une population à bas risque.

Méthodes. - Nous avons réalisé une étude de cohorte rétrospective en population dans les dix maternités du réseau MYPA de 2009 à 2015. Nous avons sélectionné une population à bas risque en ne conservant que les groupes 1 et 3 de la classification de Robson. Le critère de jugement principal était l'accouchement par césarienne. Les facteurs organisationnels étudiés ont regroupé les caractéristiques structurelles des maternités (universitaire ou non, public ou privée, nombre d'accouchement annuel, unité de soins intensifs maternels, unité de soins intensifs néonatals, nombre de salle de naissance) et l'organisation de l'équipe soignante (nombre de sages-femmes et d'obstétriciens, mode de garde, charge de travail et réalisation d'une revue de morbi-mortalité). Nous avons réalisé une analyse multiniveau à intercept aléatoire, univariée puis multivariée en retenant les variables ayant un $p<0,20$.

Résultats. - Notre population d'étude a inclus 64100 femmes. Le taux de césarienne a varié de 5,5 à $11,3 \%$ parmi les 10 maternités. En analyse univariée, les variables organisationnelles significativement associées à la réalisation d'une césarienne étaient le statut de la maternité (centre hospitalier universitaire, centre hospitalier public non universitaire et établissement privé) et la réalisation d'une revue de morbi-mortalité. Cependant, après ajustement, aucun de ces facteurs organisationnels n'était significativement associé à la réalisation d'une césarienne. Lorsque l'analyse multivariée a été restreinte au sous-groupe des nullipares, le statut privé de la maternité était significativement associé à un taux plus élevé de césariennes $(O R=1,39[1,09-1,76])$. De même, la probabilité de césarienne était plus importante lorsque le nombre d'accouchement par salle de naissance augmentait ( $\mathrm{OR}=1,15[1,01$ $1,31]$ ). Pour le sous-groupe des multipares, aucune variable n'était significativement associée à la réalisation d'une césarienne.

Conclusion. - Dans notre population globale à bas risque, aucun facteur organisationnel n'apparaissait associé à une augmentation du taux de césarienne. En revanche, dans la population des nullipares à bas risque, le statut privé du centre et un nombre élevé d'accouchement par salle de naissance étaient associés à un plus grand nombre de césariennes. Augmenter le nombre de salles de naissance pourrait donc être une piste pour tenter de réduire le nombre de césariennes. Les travaux à venir devraient également chercher à identifier les facteurs spécifiques permettant de réduire les différences de taux de césarienne entre maternités privées et publiques.
\end{abstract}

(c) 2018 Elsevier Masson SAS. Tous droits réservés.

\footnotetext{
* Auteur correspondant.

Adresse e-mail : prozenberg@chi-poissy-st-germain.fr (P. Rozenberg).
} 


\begin{abstract}
A B S T R A C T
Objective. - Huge differences in cesarean delivery rate exist between maternities in a same region. In recent years, the cesarean delivery rate has increased in the low-risk population. The objective of this study was to assess the impact of organizational factors on the cesarean delivery occurence in a low-risk population. Methods. - We performed a population-based cohort study in the MYPA perinatal network from 2009 to 2015. A low-risk population was selected, keeping only groups 1 and 3 of the Robson classification. The studied organizational factors included the structural characteristics of maternity (academic or not public or private, number of annual births, maternal intensive care unit, neonatal intensive care unit, number of delivery rooms) and the organization of the health care team (number of midwives and obstetricians, on call duty, workload and implementation of a morbi-mortality review). We used a logistic multilevel model, based on random center effect, for both univariable and multivariable analysis. Results. - Our study population included 64,100 women. The cesarean delivery rate ranged from 5.5 to $11.3 \%$ among the 10 maternities. In univariate analysis, the organizational variables significantly associated with the cesarean delivery rate were maternity status (university hospital, non-university public hospital and private hospital) and the implementation of a morbi-mortality review. However, after adjustment, none of these organizational factors were significantly associated with the cesarean delivery occurence. When multivariate analysis was restricted to the nulliparous subgroup, the private status of maternity was significantly associated with a higher rate of cesarean deliveries ( $\mathrm{OR}=1.39$ [1.09-1.76]). Also, the probability of cesarean delivery was higher when the number of births by delivery room increased ( $\mathrm{OR}=1.15$ [1.01-1.31]). For the multiparous subgroup, no variable was significantly associated with cesarean delivery occurence.

Conclusion. - In our global low-risk population, no organizational factors appeared to be associated with an increase in cesarean delivery rate. On the other hand, in the low-risk nulliparous population, the private status of the maternity and a high number of births by delivery room were associated with more cesarean deliveries. Increasing the number of delivery rooms could be a way to reduce the number of cesarean deliveries. Future researches should also try to identify specific factors that can reduce differences in cesarean delivery rates between private and public maternities.
\end{abstract}

(c) 2018 Elsevier Masson SAS. All rights reserved.

\section{Introduction}

La césarienne est l'intervention chirurgicale la plus pratiquée dans le monde. Or, il existe des différences considérables de taux de césariennes entre les pays, les régions d'un même pays, et même les maternités d'une même région. Le taux de césarienne varie par exemple de $17 \%$ [1] en Finlande à $32 \%$ aux États-Unis [2] et même $56 \%$ au Brésil [3]. En France, le taux de césarienne est de 20,4\% [4]. Au sein du réseau de périnatalité MYPA (maternité en Yvelines et périnatalité active), des variations importantes du taux global de césarienne ont été rapportées entre les 10 maternités du réseau allant de 18,4 à 29,6 \% [5]. Il est donc important et utile de comprendre les éléments contribuant à cette grande variabilité.

Dans ce but, l'Organisation mondiale de la santé (OMS) conseille de comparer les différentes maternités entre elles [6] en utilisant la classification de Robson [7-9]. Cette classification est totalement inclusive et basée sur les caractéristiques obstétricales des patientes. Elle comprend dix groupes, qui sont mutuellement exclusifs. Les groupes sont répartis suivant le nombre de fœtus, la parité, la présentation fœtale, le mode de début de travail, le terme à l'accouchement et l'éventuel antécédent de césarienne. Un avantage intéressant de la classification de Robson est de sélectionner facilement une population à bas risque.

Le taux de césarienne a augmenté ces dernières années dans la population à bas risque des nullipares à terme ayant un seul fœtus en présentation céphalique (groupe 1 de Robson). Cette augmentation du nombre de césarienne induit une augmentation de la contribution du groupe à bas risque au taux global de césarienne [10].

Plusieurs facteurs contribuant à l'augmentation du taux de césarienne ont été identifiés, notamment, les caractéristiques maternelles médicales, obstétricales et socio-économiques, le choix de la patiente et de l'obstétricien [11-15]. Cependant, il est peu probable que ces facteurs expliquent à eux seuls des variations considérables du taux de césarienne entre maternités $[5,16]$, notamment dans des populations à bas risque obstétrical.
C'est pourquoi l'objectif de cette étude était d'étudier l'impact des facteurs organisationnels sur le taux de césarienne dans une population à bas risque.

\section{Méthodes}

Nous avons réalisé une étude de cohorte rétrospective en population sur tous les accouchements ayant eu lieu dans les dix maternités du réseau de périnatalité maternités en Yvelines et périnatalité active (MYPA) de janvier 2009 à décembre 2015.

Les données ont été extraites de la base de données CoNaissance 78. Ce programme repose sur l'exploitation du premier certificat de santé (PCS) complété par un certificat complémentaire ainsi qu'un certificat d'enfant mort-né, en collaboration avec le Conseil Départemental des Yvelines et l'unité Inserm EPOPé. Les principaux résultats issus de la base de donnée CoNaissance 78 ont récemment été publiés et montrent que le taux d'exhaustivité des PCS enregistrés dans les maternités du réseau MYPA par rapport à l'État Civil est de 99,1\% [5]. Nous avons classé tous les accouchements de la période d'étude selon la classification de Robson. Nous avons ensuite sélectionné une population à bas risque en ne conservant que les groupes 1 (nullipares à terme, en présentation céphalique, en travail spontané avec un seul fœtus) et 3 (multipares à terme, en présentation céphalique, en travail spontané avec un seul fotus, sans antécédent de césarienne).

Le critère de jugement principal était l'accouchement par césarienne.

Les facteurs organisationnels étudiés ont regroupé les caractéristiques structurelles des maternités et l'organisation de l'équipe soignante. Ces caractéristiques ont été sélectionnées à partir des données de la littérature [17-20]. Les caractéristiques structurelles recueillies étaient le statut des maternités (centre hospitalieruniversitaire, centre hospitalier public non universitaire et établissement privé), le nombre d'accouchement annuel et la composition du plateau technique (unité de soins intensifs 
maternels, unité de soins intensifs néonatals, nombre de salle de naissance). Les caractéristiques recueillies pour définir l'organisation de l'équipe soignante étaient, le mode de garde de l'anesthésiste et de l'obstétricien, la réalisation d'une revue de morbimortalité (RMM) et la charge de travail des médecins et sagesfemmes, modélisée par le ratio du nombre annuel d'accouchement sur le nombre de sages-femmes de garde en salle de naissance d'une part et le nombre d'obstétricien prenant des gardes dans la maternité d'autre part.

Les variables quantitatives ont été transformées en variables catégorielles. Nous avons réalisé une régression logistique en utilisant un modèle multiniveau à intercept aléatoire pour l'analyse univariée et l'analyse multivariée car nous avions comme hypothèse que nos données étaient corrélées au niveau du centre. L'utilisation d'un modèle multiniveau permettait de prendre en compte cette corrélation due aux données hiérarchiques et d'obtenir des odds ratio plus précis. Le premier niveau de ce modèle multiniveau correspondait aux données individuelles (femmes) et le deuxième niveau à la maternité. Nous avons dans un premier temps réalisé une analyse univariée et calculé les odds ratio avec leurs intervalles de confiance des facteurs potentiellement associés aux césariennes. Nous avons ensuite fait une analyse multivariée avec toutes les variables ayant un $p<0,20$ et vérifié qu'il n'y avait pas d'interaction entre les variables du modèle. L'analyse multivariée a été réalisée en ajustant sur les variables obstétricales et socio-économiques significatives (âge maternel, niveau d'études, statut professionnel du père).

L'analyse multivariée a été réalisée sur les données complètes et nous avons estimés les odds ratios ajustés associés à la survenue d'une césarienne pendant le travail.

L'analyse a été réalisée dans un premier temps sur la population globale puis nous avons fait une analyse en sous-groupes afin d'étudier plus spécifiquement les facteurs associés au risque de césarienne parmi les nullipares et parmi les multipares.

L'analyse statistique a été réalisée avec $\mathrm{R}$ Studio, version 1.0.136.

\section{Résultats}

Durant la période d'étude, 124945 accouchements ont été enregistrés. Notre population d'étude correspondant aux groupes 1 et 3 de Robson a inclus 64100 femmes (Fig. 1). Dans cette population à bas risque, 29423 femmes étaient nullipares (46\%). Le taux de césarienne a varié de $5,5 \%$ à $11,3 \%$ parmi les 10 maternités (Fig. 2), allant de 9,0 à 20,0\% parmi les nullipares et de 2,0 à 6,0\% parmi les multipares. Nous avons dans un premier temps décrit les variables organisationnelles au niveau de chaque maternité (Tableau 1).

En analyse univariée, les variables organisationnelles significativement associées au taux de césarienne étaient le statut de la maternité (centre hospitalier-universitaire, centre hospitalier public non universitaire et établissement privé) et la réalisation d'une RMM (Tableau 2). Après ajustement sur l'âge maternel, la parité, le niveau d'étude, le statut familial (en couple ou célibataire) et le statut professionnel de la patiente et de son conjoint, aucun de ces facteurs organisationnels n'étaient significativement associés au taux de césarienne (Tableau 3).

Lorsque l'analyse univariée a été restreinte au sous-groupe des nullipares, les variables organisationnelles significativement associées au risque de césarienne étaient le statut de la maternité, la réalisation d'une RMM, les ratios nombre d'accouchement par nombre de sage-femme, nombre d'accouchement par nombre de salle de naissance et nombre d'accouchement par nombre d'obstétricien (Tableau 4). Les variables " ratio d'accouchement par salle de naissance », " ratio d'accouchement par obstétricien » et " ratio d'accouchement par sage-femme " étant fortement corrélées entre elles, nous avons donc réalisé trois modèles pour étudier en analyse multivariée chacune de ces variables séparément (Tableau 5).

Le statut privé de la maternité était significativement associé à un nombre plus élevé de césariennes (OR = 1,39 [1,09-1,76]). De même, la probabilité de césarienne était plus importante lorsque le ratio accouchement par salle de naissance augmentait $(\mathrm{OR}=1,15$

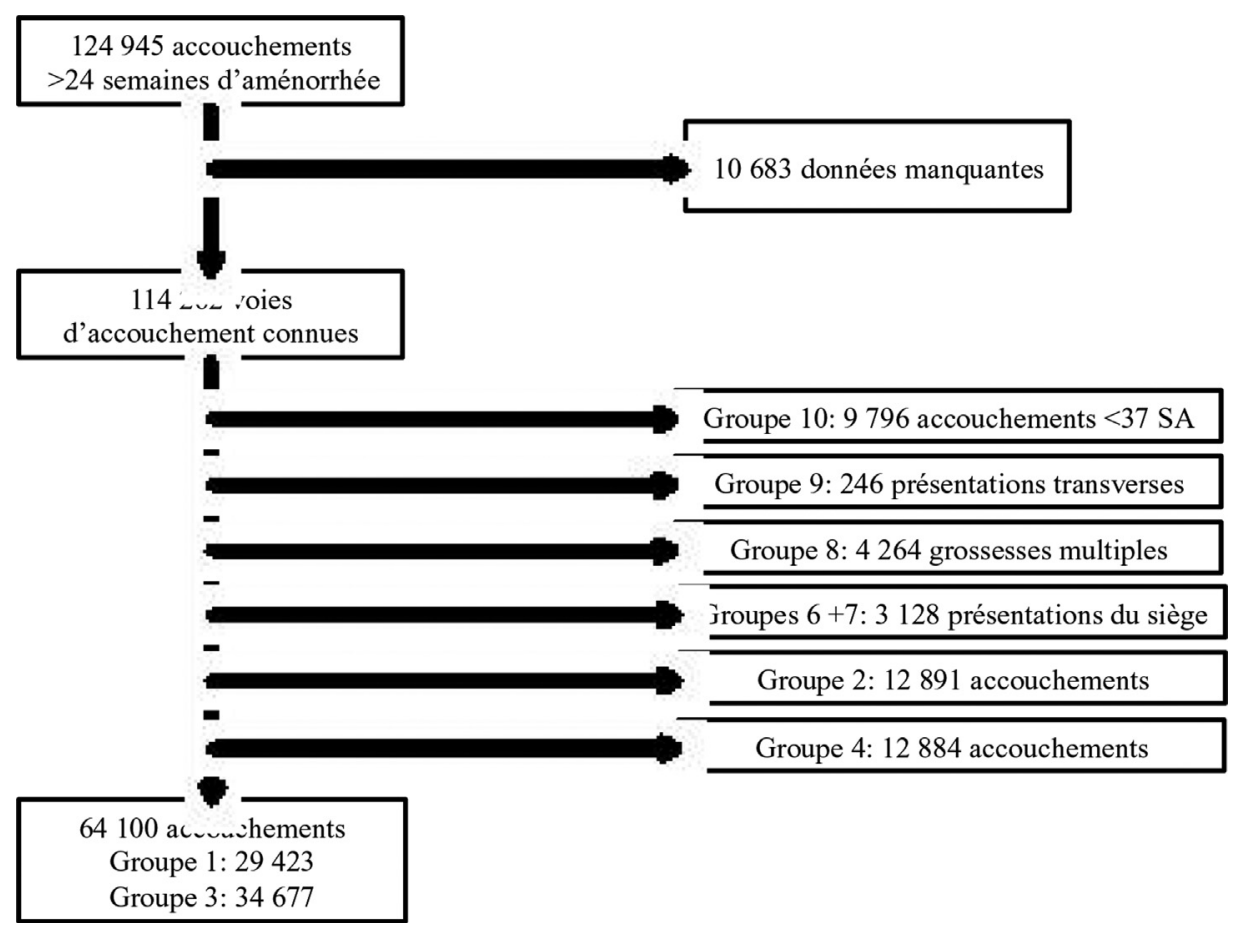

Fig. 1. Diagramme de flux. 


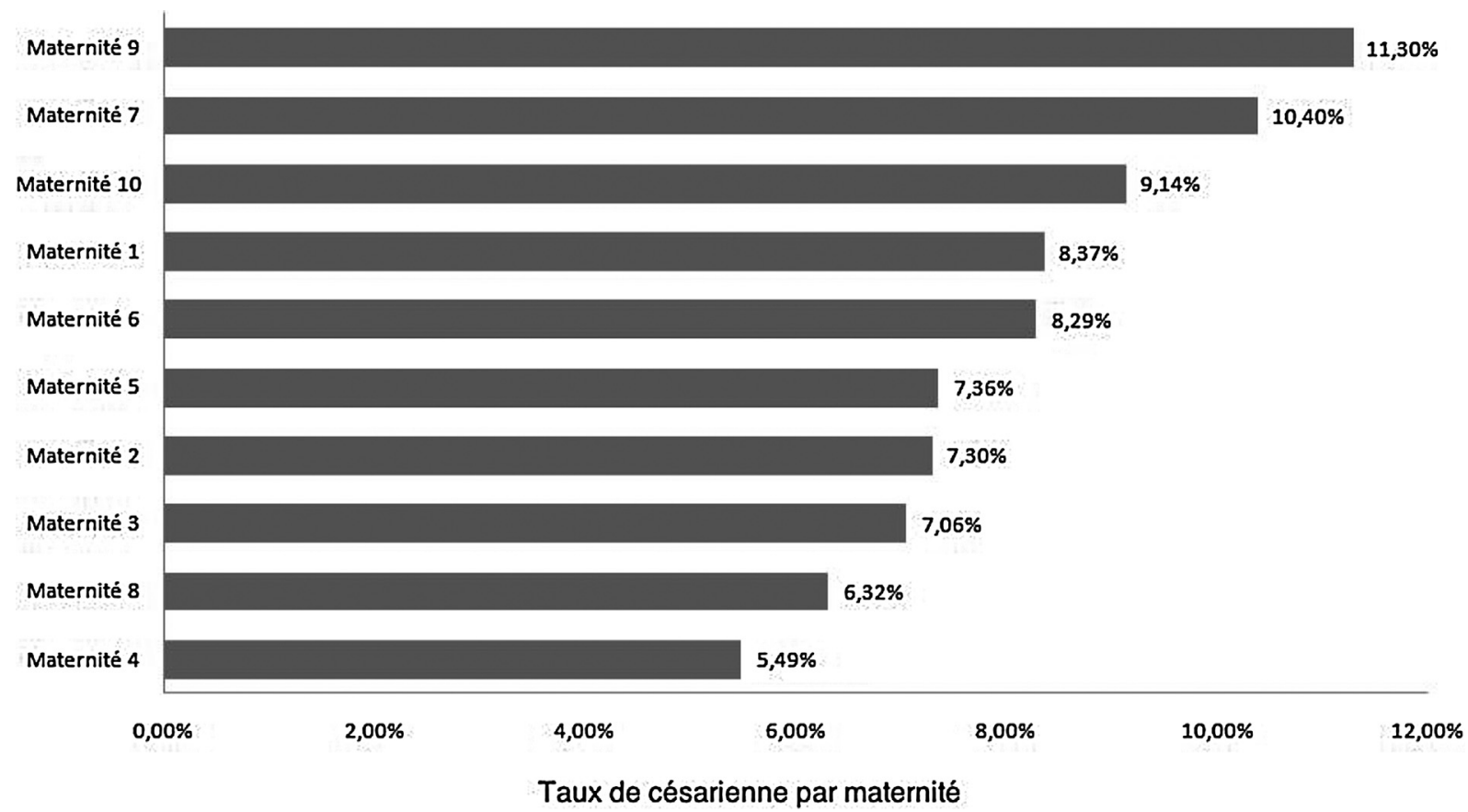

Fig. 2. Graphique représentant le taux de césarienne par maternité sur la population d'étude.

Tableau 1

Description de la population.

\begin{tabular}{|c|c|c|c|c|c|c|c|c|c|c|}
\hline & Maternité & Maternité & Maternité & Maternité & Maternité & Maternité & Maternité & Maternité & Maternité & Maternité \\
\hline & 1 & 2 & 3 & 4 & 5 & 6 & 7 & 8 & 9 & 10 \\
\hline \multicolumn{11}{|l|}{ Statut } \\
\hline Centre hospitalier non universitaire & & + & + & + & + & & & & & \\
\hline Centre hospitalo-universitaire & + & & & & & & & & & \\
\hline Centre privé & & & & & & + & + & + & + & + \\
\hline \multicolumn{11}{|l|}{ Soins intensifs néonataux } \\
\hline Non & & + & & + & + & + & + & + & + & + \\
\hline Oui & + & & + & & & & & & & \\
\hline Nb. total d'accouchement & 16598 & 3904 & 9136 & 5599 & 7875 & 4757 & 2175 & 3055 & 4101 & 6850 \\
\hline \multicolumn{11}{|l|}{ Nb. d'accouchement par an } \\
\hline$<1500$ & & + & & + & & + & + & + & + & \\
\hline $1500-2500$ & & & & & + & & & & & \\
\hline$>2500$ & + & & + & & & & & & & + \\
\hline \multicolumn{11}{|l|}{ Ratio accouchement par salle de travail } \\
\hline Quartile 1 [102-335] & & & & & & + & + & + & + & \\
\hline Quartile $2+3$ [336-541] & & + & + & + & + & & & & & + \\
\hline Quartile 4 [542-583] & + & & & & & & & & & \\
\hline Soins intensifs maternels & & & & & & & & & & + \\
\hline Non & & & & & & + & + & & & + \\
\hline Oui & + & + & + & + & + & & & + & + & \\
\hline \multicolumn{11}{|l|}{ Présence de l'obstétricien } \\
\hline Garde de 24 heures & + & + & + & + & + & & & + & + & + \\
\hline Astreinte & & & & & & + & + & & & \\
\hline \multicolumn{11}{|l|}{ Présence de l'anesthésiste } \\
\hline Garde de 24 heures dans la maternité & + & & + & & + & & & & & + \\
\hline Garde de 24 heures dans l'hôpital & & + & & + & & + & + & + & + & \\
\hline \multicolumn{11}{|l|}{ Ratio accouchement par sage-femme } \\
\hline Quartile 1 [308-767] & & & & + & & + & + & & + & \\
\hline Quartile $2+3$ [768-1138] & + & + & & & + & & & + & & + \\
\hline Quartile 4 [1139-1387] & & & + & & & & & & & \\
\hline \multicolumn{11}{|l|}{ Ratio accouchement par obstétricien } \\
\hline Quartile 1 [77-166] & & & & & + & & & + & & \\
\hline Quartile 2+3 [167-714] & & + & + & + & & + & + & & + & + \\
\hline Quartile 4 [715-779] & + & & & & & & & & & \\
\hline \multicolumn{11}{|l|}{$R M M$} \\
\hline Non & & & & & & + & & & + & \\
\hline Oui & + & + & + & + & + & & + & + & & + \\
\hline
\end{tabular}

Pour citer cet article : Duvillier C, et al. Facteurs organisationnels associés à la réalisation d'une césarienne dans une population à bas risque. Gynécologie Obstétrique Fertilité \& Sénologie (2018), https://doi.org/10.1016/j.gofs.2018.09.005 
Tableau 2

Variables organisationnelles associées au taux de césariennes - analyse univariée avec un modèle à intercept aléatoire à effet centre.

\begin{tabular}{|c|c|c|c|}
\hline Variables & OR & IC95 \% & $p$ \\
\hline Statut & & & 0,02 \\
\hline Centre hospitalier non universitaire & 1 & & \\
\hline Centre hospitalo-universitaire & 1,26 & {$[0,89 ; 1,77]$} & \\
\hline Centre privé & 1,36 & {$[1,09 ; 1,69]$} & \\
\hline Soins intensifs néonataux & & & 0,82 \\
\hline Non & 1 & & \\
\hline Oui & 0,96 & {$[0,67 ; 1,37]$} & \\
\hline Nombre d'accouchement par an & & & 0,66 \\
\hline $1500-2500$ & 1 & & \\
\hline$<1500$ & 1,06 & {$[0,94 ; 1,19]$} & \\
\hline$>2500$ & 1 & {$[0,84 ; 1,18]$} & \\
\hline Ratio accouchement par salle de travail & & & 0,26 \\
\hline Quartile 2+3 [336-541] & 1 & & \\
\hline Quartile 1 [102-335] & 1,03 & {$[0,95 ; 1,12]$} & \\
\hline Quartile 4 [542-583] & 1,07 & {$[0,99 ; 1,17]$} & \\
\hline Soins intensifs maternels & 0,91 & {$[0,68 ; 1,23]$} & 0,54 \\
\hline Présence de l'obstétricien & & & 0,87 \\
\hline Garde de 24 heures & 1 & & \\
\hline Astreinte & 1,03 & {$[0,73 ; 1,46]$} & \\
\hline Présence de l'anesthésiste & & & 0,98 \\
\hline Garde de 24 heures dans la maternité & 1 & & \\
\hline Garde de 24 heures dans l'hôpital & $>0,99$ & {$[0,76 ; 1,33]$} & \\
\hline Ratio accouchement par sage-femme & & & 0,06 \\
\hline Quartile 2+3 [768-1138] & 1 & & \\
\hline Quartile 1 [308-767] & 1,02 & {$[0,95 ; 1,09]$} & \\
\hline Quartile 4 [1139-1387] & 0,89 & {$[0,79 ; 0,99]$} & \\
\hline Ratio accouchement par obstétricien & & & 0,25 \\
\hline Quartile 2+3 [167-714] & 1 & & \\
\hline Quartile 1 [77-166] & 1,05 & {$[0,94 ; 1,17]$} & \\
\hline Quartile 4 [715-779] & 1,09 & {$[0,98 ; 1,22]$} & \\
\hline RMM & 0,76 & {$[0,57 ; 1,00]$} & 0,05 \\
\hline
\end{tabular}

Tableau 3

Variables organisationnelles associées au taux de césariennes - analyse multivariée avec un modèle à intercept aléatoire à effet centre.

\begin{tabular}{llll}
\hline Variables & OR & IC95 \% & $p$ value \\
\hline $\begin{array}{l}\text { Statut } \\
\quad \text { Centre hospitalier non universitaire }\end{array}$ & 1 & & 0,16 \\
$\quad$ Centre hospitalo-universitaire & 1,26 & {$[0,91 ; 1,75]$} & 0,28 \\
$\quad$ Centre privé & 1,29 & {$[1,00 ; 1,65]$} & \\
RMM & 0,90 & {$[0,67 ; 1,21]$} & 0,50 \\
Ratio accouchement par sage-femme & & & 0,28 \\
$\quad$ Quartile 2+3 [768-1138] & 1 & & \\
$\quad$ Quartile 1 [308-767] & 1,07 & {$[0,99 ; 1,16]$} & \\
$\quad$ Quartile 4 [1139-1387] & 1,00 & {$[0,86 ; 1,15]$} & \\
\end{tabular}

$[1,01-1,31])$. La variance inter maternités était de 0,05 et le pourcentage de variance expliquée par le niveau centre était de $0,06 \%$. La part de variance expliquée par nos modèles restait donc faible indiquant qu'une part importante des facteurs impliqués dans les variations du taux de césarienne reste inexpliquée.

Pour le sous-groupe des multipares, aucune variable n'était significativement associée à la variation du nombre de césarienne (Tableau 6).

\section{Discussion}

Notre étude montre qu'au sein d'une population à bas risque, le taux de césarienne variait de manière importante, passant du simple au double suivant la maternité. Cependant, en considérant la population globale, aucune variable organisationnelle, qu'il s'agisse des caractéristiques structurelles des maternités ou de l'organisation de l'équipe soignante, n'était associée significativement à la survenue d'une césarienne. En revanche, le statut de la maternité et le ratio d'accouchement par salle de naissance étaient
Tableau 4

Variables organisationnelles associées au taux de césariennes - analyse univariée sur le sous-groupe des nullipares avec un modèle à intercept aléatoire à effet centre.

\begin{tabular}{|c|c|c|c|}
\hline Variables & OR & IC95 \% & $p$ \\
\hline Statut & & & 0,14 \\
\hline Centre hospitalier non universitaire & 1 & & \\
\hline Centre hospitalo-universitaire & 1,13 & {$[0,74 ; 1,71]$} & \\
\hline Centre privé & 1,31 & {$[1,00 ; 1,70]$} & \\
\hline Soins intensifs néonataux & & & 0,8 \\
\hline Non & 1 & & \\
\hline Oui & 0,95 & {$[0,66 ; 1,37]$} & \\
\hline Nombre d'accouchement par an & & & 0,81 \\
\hline $1500-2500$ & 1 & & \\
\hline$<1500$ & 0,97 & {$[0,85,1,12]$} & \\
\hline$>2500$ & 0,94 & {$[0,78 ; 1,14]$} & \\
\hline Ratio accouchement par salle de travail & & & 0,1 \\
\hline Quartile 2+3 [336-541] & 1 & & \\
\hline Quartile 1 [102-335] & 1 & {$[0,91 ; 1,10]$} & \\
\hline Quartile 4 [542-583] & 1,09 & {$[0,99 ; 1,21]$} & \\
\hline Soins intensifs maternels & 0,97 & {$[0,70 ; 1,33]$} & 0,83 \\
\hline Présence de l'obstétricien & & & 0,87 \\
\hline Garde de 24 heures & 1 & & \\
\hline Astreinte & 0,97 & {$[0,67 ; 1,41]$} & \\
\hline Présence de l'anesthésiste & & & 0,76 \\
\hline Garde de 24 heures dans la maternité & 1 & & \\
\hline Garde de 24 heures dans l'hôpital & 1,05 & {$[0,78 ; 1,41]$} & \\
\hline Ratio accouchement par sage-femme & & & 0,12 \\
\hline Quartile 2+3 [768-1138] & 1 & & \\
\hline Quartile 1 [308-767] & 1,03 & {$[0,96 ; 1,12]$} & \\
\hline Quartile 4 [1139-1387] & 0,9 & {$[0,90 ; 1,02]$} & \\
\hline Ratio accouchement par obstétricien & & & 0,09 \\
\hline Quartile 2+3 [167-714] & 1 & & \\
\hline Quartile 1 [77-166] & 1,06 & {$[0,93 ; 1,21]$} & \\
\hline Quartile 4 [715-779] & 1,15 & {$[1,01 ; 1,30]$} & \\
\hline$R M M$ & 0,72 & {$[0,53 ; 0,97]$} & 0,03 \\
\hline
\end{tabular}

significativement associés au taux de césarienne lorsque seule la population des nullipares était considérée.

Parmi les nullipares, le quartile correspondant au plus grand nombre d'accouchement par salle de naissance était, en effet, associé à une augmentation du nombre de césariennes. Cette variable reflétant l'occupation des salles de naissance, les variations de flux de nombre d'accouchement par salle semblent donc avoir un impact sur le taux de césarienne. Une activité importante pourrait donc influencer les pratiques des obstétriciens et conduire plus facilement à une décision de césarienne. Ce résultat n'est pas retrouvé chez les multipares. Le travail spontané de ce groupe de patientes étant plus rapide, il n'est pas surprenant que les variables organisationnelles n'aient pas ou moins d'influence.

En revanche, quand la disponibilité du personnel soignant et le mode de garde sont considérés, aucune des variables n'étaient associée significativement à la survenue d'une césarienne, contrairement à ce qui avait été précédemment décrit dans d'autres pays [21].

De plus, la charge de travail des sages-femmes n'était pas, dans notre population, associée aux variations de taux de césariennes. Ces résultats contradictoires d'autres études [22-25] peuvent s'expliquer par l'existence de recommandations éditées par la Haute Autorité de Santé. En effet les pratiques sont standardisées en France, et suivant le nombre d'accouchement annuel d'une maternité, le nombre de sages-femmes en salle de naissance et de pré-travail ainsi que le mode de garde de l'équipe médical est défini. Enfin, comme d'autres études l'avaient déjà montré, le nombre annuel d'accouchements et le type de maternité défini par la présence ou non d'une USI néonatale n'étaient pas associés à une augmentation du nombre de césarienne $[17,26]$.

Nous avons utilisé la classification de Robson pour sélectionner notre population d'étude à bas risque. En effet, la plupart des 
Tableau 5

Variables organisationnelles associées au taux de césariennes - analyse multivariée sur le sous-groupe des nullipares.

\begin{tabular}{|c|c|c|c|c|c|c|}
\hline \multirow[b]{2}{*}{ Variables } & \multicolumn{2}{|c|}{ Modele 1} & \multicolumn{2}{|c|}{ Modele 2} & \multicolumn{2}{|c|}{ Modele 3} \\
\hline & OR & IC95 \% & OR & IC95 \% & OR & IC95 \% \\
\hline \multicolumn{7}{|l|}{ Statut } \\
\hline Centre hospitalier non universitaire & 1 & & 1 & & 1 & \\
\hline Centre hospitalo-universitaire & 1,17 & {$[0,85 ; 1,62]$} & 1,21 & {$[0,88 ; 1,67]$} & 1,09 & {$[0,74 ; 1,59]$} \\
\hline Centre privé & 1,39 & {$[1,09 ; 1,76]$} & 1,31 & {$[1,02 ; 1,68]$} & 1,35 & {$[1,05 ; 1,74]$} \\
\hline \multicolumn{7}{|l|}{ Ratio accouchement par salle de travail } \\
\hline Quartile $2+3$ [336-541] & 1 & & & & & \\
\hline Quartile 1 [102-335] & 0,98 & {$[0,87 ; 1,09]$} & & & & \\
\hline Quartile 4 [542-583] & 1,15 & {$[1,01 ; 1,31]$} & & & & \\
\hline \multicolumn{7}{|l|}{ Ratio accouchement par sage-femme } \\
\hline Quartile 2+3 [768-1138] & & & 1 & & & \\
\hline Quartile 1 [308-767] & & & 1,09 & {$[0,99 ; 1,20]$} & & \\
\hline Quartile 4 [1139-1387] & & & 1,02 & {$[0,87 ; 1,19]$} & & \\
\hline \multicolumn{7}{|l|}{ Ratio accouchement par obstétricien } \\
\hline Quartile $2+3$ [167-714] & & & & & 1 & \\
\hline Quartile 1 [77-166] & & & & & 1,04 & {$[0,89 ; 1,22]$} \\
\hline Quartile 4 [715-779] & & & & & 1,16 & {$[1,00 ; 1,36]$} \\
\hline$R M M$ & 0,93 & {$[0,72 ; 1,22]$} & 0,9 & {$[0,67 ; 1,21]$} & 0,99 & {$[0,73 ; 1,33]$} \\
\hline \multicolumn{7}{|l|}{ Âge maternel } \\
\hline $20-35$ ans & 1 & & 1 & & 1 & \\
\hline$<20$ ans & 1,41 & {$[1,30 ; 1,55]$} & 1,41 & {$[1,30 ; 1,55]$} & 1,42 & {$[1,30 ; 1,55]$} \\
\hline$>35$ ans & 1,49 & {$[1,12 ; 1,96]$} & 1,48 & {$[1,12 ; 1,96]$} & 1,48 & {$[1,12 ; 1,96]$} \\
\hline \multicolumn{7}{|l|}{ Niveau d'études } \\
\hline Études supérieures & 1 & & 1 & & 1 & \\
\hline École primaire et secondaire & 1,35 & {$[1,17 ; 1,54]$} & 1,35 & {$[1,17 ; 1,54]$} & 1,34 & {$[1,17 ; 1,54]$} \\
\hline Niveau bac & 1,37 & {$[1,24 ; 1,52]$} & 1,37 & {$[1,24 ; 1,52]$} & 1,37 & {$[1,24 ; 1,52]$} \\
\hline \multicolumn{7}{|l|}{ Statut professionnel du père } \\
\hline Actif & 1 & & 1 & & 1 & \\
\hline Chômage & 1,08 & {$[0,88 ; 1,31]$} & 1,08 & {$[0,88 ; 1,31]$} & 1,08 & {$[0,88 ; 1,31]$} \\
\hline Sans emploi & 1,09 & {$[0,92 ; 1,30]$} & 1,09 & {$[0,92 ; 1,30]$} & 1,09 & {$[0,92 ; 1,30]$} \\
\hline
\end{tabular}

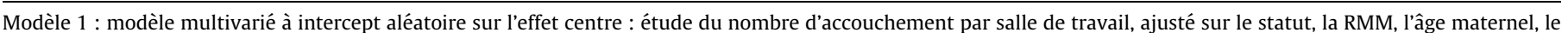

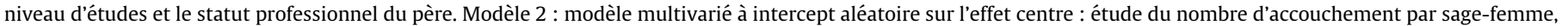

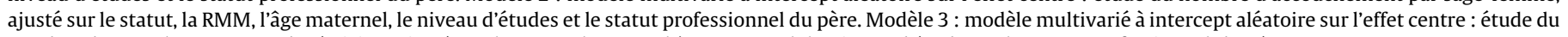
nombre d'accouchement par obstétricien, ajusté sur le statut, la RMM, l'âge maternel, le niveau d'études et le statut professionnel du père.

\section{Tableau 6}

Variables organisationnelles associées au taux de césariennes - analyse univariée sur le sous-groupe des multipares avec un modèle à intercept aléatoire à effet centre.

\begin{tabular}{|c|c|c|c|}
\hline Variables & OR & IC95 \% & $p$ \\
\hline Statut & & & 0,18 \\
\hline Centre hospitalier non universitaire & 1 & & \\
\hline Centre hospitalo-universitaire & 1,41 & {$[0,89 ; 2,22]$} & \\
\hline Centre privé & 1,28 & {$[0,95 ; 1,73]$} & \\
\hline Soins intensifs néonataux & & & 0,72 \\
\hline Non & 1 & & \\
\hline Oui & 1,08 & {$[0,72 ; 1,60]$} & \\
\hline Nombre d'accouchement par an & & & 0,48 \\
\hline $1500-2500$ & 1 & & \\
\hline$<1500$ & 1,11 & {$[0,91,1,36]$} & \\
\hline$>2500$ & 1,1 & {$[0,86 ; 1,41]$} & \\
\hline Ratio accouchement par salle de travail & & & 0,57 \\
\hline Quartile 2+3 [336-541] & 1 & & \\
\hline Quartile 1 [102-335] & 1,07 & {$[0,92 ; 1,25]$} & \\
\hline Quartile 4 [542-583] & 1 & {$[0,86 ; 1,17]$} & \\
\hline Soins intensifs maternels & 0,96 & {$[0,67 ; 1,37]$} & 0,81 \\
\hline Présence de l'obstétricien & & & 0,85 \\
\hline Garde de 24 heures & 1 & & \\
\hline Astreinte & 0,96 & {$[0,62 ; 1,49]$} & \\
\hline Présence de l'anesthésiste & & & 0,49 \\
\hline Garde de 24 heures dans la maternité & 1 & & \\
\hline Garde de 24 heures dans l'hôpital & 0,89 & {$[0,64 ; 1,24]$} & \\
\hline Ratio accouchement par sage-femme & & & 0,56 \\
\hline Quartile $2+3$ [768-1138] & 1 & & \\
\hline Quartile 1 [308-767] & 0,98 & {$[0,86 ; 1,11]$} & \\
\hline Quartile 4 [1139-1387] & 0,9 & {$[0,74 ; 1,09]$} & \\
\hline Ratio accouchement par obstétricien & & & 0,77 \\
\hline Quartile $2+3$ [167-714] & 1 & & \\
\hline Quartile 1 [77-166] & 1,02 & {$[0,84 ; 1,24]$} & \\
\hline Quartile 4 [715-779] & 1,07 & {$[0,88 ; 1,30]$} & \\
\hline$R M M$ & 0,81 & {$[0,56 ; 1,18]$} & 0,28 \\
\hline
\end{tabular}

femmes ayant une pathologie médicale ou obstétricale ayant habituellement un déclenchement artificiel du travail ou une césarienne programmée n'appartiennent donc pas aux groupes 1 et 3 de la classification de Robson. De plus, les facteurs de risque obstétricaux connus, à savoir la parité, le nombre de fœetus, la présentation fœtale, le mode de début de travail, l'antécédent de césarienne, la prématurité, interviennent dans cette classification pour définir les différents groupes. L'intérêt d'étudier cette population à bas risque était de supposer que ces patientes avaient a priori un risque faible de césarienne et donc que les variations du taux de césarienne entre les maternités devaient être minimales. Ce choix de population avait déjà été publié, montrant que cette population à bas risque représentait la majeure partie des parturientes [27]. De plus, grâce à l'utilisation de la classification de Robson, les principales variables obstétricales des parturientes étaient incluses dans notre analyse. Nous avons également inclus dans notre modèle l'âge maternel, facteur de risque établi de césarienne. Cependant, nous n'avons pas pu inclure, compte-tenu de la nature de notre base de données, l'indice de masse corporelle (IMC) des patientes, autre facteur de risque important [26,28,29].

La part de la variance expliquée étant très faible, d'autres facteurs organisationnels, non pris en compte dans notre modèle influencent très certainement le taux de césarienne comme l'existence de protocole, la discussion prospective ou rétrospective collégiale des dossiers, la réalisation de séances de simulation, elearning. De même l'absence d'inclusion de facteurs médicaux et l'IMC doivent contribuer à cette faible part de la variabilité.

De même, la contribution du facteur " choix de la patiente " n'a pas pu être étudié dans notre modèle. Toutefois, il est très faible en France puisque le taux de césariennes électives pour désir maternel parmi les nullipares est inférieur à $1 \%$ [30]. 
L'originalité de notre travail était d'inclure dans le même modèle les variables organisationnelles, obstétricales et socioéconomiques, afin d'essayer de comprendre les éléments qui font varier le taux de césarienne avec autant d'importance. Nous avons disposé d'une grande population d'étude, soit 64100 patientes sur sept années consécutives. De plus, la quasi-totalité des accouchements du réseau étaient inclus dans cette base de donnée puisque la base CoNaissance 78 a un taux d'exhaustivité de $99,1 \%$. Notre étude regroupait 10 maternités, 5 étant publiques et 5 étant privées. Cependant ces dix maternités appartenaient à un seul réseau de périnatalité. De plus, une maternité regroupait le plus grand nombre d'accouchement et était le seul centre hospitalier universitaire et le seul disposant d'une réanimation néonatale. Ce centre avait un poids important dans l'étude et le réseau MYPA pourrait différer des autres réseaux de périnatalité de France, limitant la validité externe de nos résultats.

Nous avons essayé de prendre en compte le niveau socioéconomique des patientes car il a été montré qu'un faible niveau socio-économique était associé à un risque accru de césarienne. Cependant nous n'avions que des proxies à disposition et nous n'avions pas de variable reflétant de manière fiable le niveau socioéconomique des patientes de notre population. Ces variables avaient toutefois déjà été utilisées dans d'autres études de périnatalité [31,32]. Enfin, une autre limite a été l'exclusion des femmes pour lesquelles la voie d'accouchement n'était pas renseignée et celles pour lesquelles les variables permettant de classer selon les groupes de Robson étaient manquantes. Cela représente respectivement 2,1 et $6,4 \%$ de la population d'étude. Nous ne pouvons donc pas éliminer un possible biais de sélection. Cependant, le faible pourcentage de données manquantes n'affecte probablement pas nos résultats.

Au total, dans notre population globale à bas risque, aucun facteur organisationnel n'apparaissait associé à une augmentation de césarienne. En revanche, il y avait toujours une variation du simple au double entre les différentes maternités. Cependant, lorsque seules les nullipares ont été prises en compte, le statut privé du centre était associé à un plus grand nombre de césariennes. Sur cette population des nullipares, un ratio élevé d'accouchement par salle de naissance semble associé à un plus grand nombre de césariennes. Ainsi, augmenter le nombre de salles de travail pourrait donc être une piste pour tenter de réduire le nombre de césariennes parmi les nullipares, et ce d'autant plus que cette population représente l'enjeu le plus important, la réduction du taux de la première césarienne permettant de réduire le taux de patientes ayant un antécédent de césarienne, population pour laquelle le risque de césarienne itérative (programmée ou en cours de travail) est particulièrement important. Les travaux à venir devraient également chercher à identifier les facteurs spécifiques permettant de réduire les différences de taux de césarienne entre maternités privées et publiques.

\section{Déclaration de liens d'intérêts}

Les auteurs déclarent ne pas avoir de liens d'intérêts.

\section{Références}

[1] Macfarlane A, Blondel B, Mohangoo A, Cuttini M, Nijhuis J, Novak Z, et al. Wide differences in mode of delivery within Europe: risk-stratified analyses of aggregated routine data from the Euro-Peristat study. BJOG 2016:123:559-68.

[2] Osterman MJK, Kochanek KD, MacDorman MF, Strobino DM, Guyer B. Annual summary of vital statistics: 2012-2013. Pediatrics 2015;135:1115-25 [Disponible sur : http://pediatrics.aappublications.org/content/early/2015/04/28 peds.2015-0434.Abstract]

[3] Betrán AP, Ye J, Moller A-B, Zhang J, Gülmezoglu AM, Torloni MR. The increasing trend in caesarean section rates: global, regional and nationa estimates: 1990-2014. PLoS ONE 2016;11:e0148343.

[4] Blondel B, Coulm B, Bonnet C, Goffinet F, Le Ray C. National Coordination Group of the National Perinatal Surveys. Trends in perinatal health in metropolita
France from 1995 to 2016: results from the French National Perinatal Surveys. J Gynecol Obstet Hum Reprod 2017;46:701-13.

[5] Lebreton E, Rozenberg P, Chalavoux K, Huart F, Cotte B, Pineau C, et al. Évaluation d'un réseau périnatal à partir des premiers certificats de santé. J Gynecol Obstet Biol Reprod 2014;43:342-50.

[6] World Health Organization. WHO statement on caesarean section rates 2015;23:149-50

[7] Betrán AP, Vindevoghel N, Souza JP, Gülmezoglu AM, Torloni MR. A systematic review of the Robson classification for caesarean section: what works, doesn't work and how to improve it. PLoS ONE 2014;9:e97769 [Bhattacharya S, editor]

[8] Torloni MR, Betran AP, Souza JP, Widmer M, Allen T, Gulmezoglu M, et al. Classifications for cesarean section: a systematic review. PLoS ONE 2011;6:e14566 [Althabe F, editor].

[9] Robson M, Hartigan L, Murphy M. Methods of achieving and maintaining an appropriate caesarean section rate. Best Pract Res Clin Obstet Gynaecol 2013;27:297-308

[10] Delbaere I, Cammu H, Martens E, Tency I, Martens G, Temmerman M. Limiting the caesarean section rate in low-risk pregnancies is key to lowering the trend of increased abdominal deliveries: an observational study. BMC Pregnancy Childbirth 2012;12:1

[11] McCourt C, Weaver J, Statham H, Beake S, Gamble J, Creedy DK. Elective cesarean section and decision-making: a critical review of the literature. Birth 2007;34:65-79

[12] Milcent C, Rochut J. Tarification hospitalière et pratique médicale : la pratique de la césarienne en France. Rev Economique 2009:60:489.

[13] Spong CY, Berghella V, Wenstrom KD, Mercer BM, Saade GR. Preventing the first cesarean delivery: summary of a joint Eunice Kennedy Shriver national institute of child health and human development, society for maternal-fetal medicine, and American college of obstetricians and gynecologists workshop. Obstet Gynecol 2012;120:1181.

[14] Robson S, Carey A, Mishra R, Dear K. Elective caesarean delivery at maternal request: a preliminary study of motivations influencing women's decisionmaking. Aust N Z J Obstet Gynaecol 2008;48:415-20.

[15] Baicker K, Buckles KS, Chandra A. Geographic variation in the appropriate use of cesarean delivery. Health Aff (Millwood) 2006;25:w355-67.

[16] Declercq E, Menacker F, MacDorman M. Maternal risk profiles and the primary cesarean rate in the United States, 1991-2002. Am J Public Health 2006;96:867-72.

[17] Coulm B, Ray C, Lelong N, Drewniak N, Zeitlin J, Blondel B. Obstetric interventions for low-risk pregnant women in France: do maternity unit characteristics make a difference? Birth 2012;39:183-91.

[18] Chaillet N, Dumont A. Evidence-based strategies for reducing cesarean section rates: a meta-analysis. Birth 2007;34:53-64.

[19] Armstrong JC, Kozhimannil KB, McDermott P, Saade GR, Srinivas SK. Comparing variation in hospital rates of cesarean delivery among low-risk women using 3 different measures. Am J Obstet Gynecol 2016;214:153-63.

[20] Snowden JM, Cheng YW, Kontgis CP, Caughey AB. The association between hospital obstetric volume and perinatal outcomes in California. Am J Obstet Gynecol 2012;207:478e1-7.

[21] Karalis E, Gissler M, Tapper A-M, Ulander V-M. Effect of hospital size and on-call arrangements on intrapartum and early neonatal mortality among low-risk newborns in Finland. Eur J Obstet Gynecol Reprod Biol 2016;198:116-9.

[22] Scott KD, Berkowitz G, Klaus M. A comparison of intermittent and continuous support during labor: a meta-analysis. Am J Obstet Gynecol 1999;180:1054-9.

[23] Zhang J, Geerts C, Hukkelhoven C, Offerhaus P, Zwart J, de Jonge A. Caesarean section rates in subgroups of women and perinatal outcomes. BJOG 2016;123:754-61

[24] Zhang J, Bernasko JW, Leybovich E, Fahs M, Hatch MC. Continuous labor support from labor attendant for primiparous women: a meta-analysis. Obstet Gynecol 1996:88:739-44.

[25] Hellerstein S, Feldman S, Duan T. Survey of obstetric care and cesarean delivery rates in Shanghai, China. Birth 2016;43:193-9 [Disponible sur : http:// onlinelibrary wiley.com/doi/10.1111/birt.12231/full, cité 18 janvier 2017].

[26] Kozhimannil KB, Law MR, Virnig BA. Cesarean delivery rates vary tenfold among US hospitals; reducing variation may address quality and cost issues. Health Aff (Millwood) 2013;32:527-35.

[27] Le Ray C, Blondel B, Prunet C, Khireddine I, Deneux-Tharaux C, Goffinet F. Stabilising the caesarean rate: which target population? BJOG 2015;122:6909.

[28] Brennan DJ, Robson MS, Murphy M, O'Herlihy C. Comparative analysis of international cesarean delivery rates using 10-group classification identifies significant variation in spontaneous labor. Am J Obstet Gynecol 2009;201:308.e1-8.

[29] Sebastião YV, Womack L, Vamos CA, Louis JM, Olaoye F, Caragan T, et al. Hospital variation in cesarean delivery rates: contribution of individual and hospital factors in Florida. Am J Obstet Gynecol 2016;214:123.e1-123.e18.

[30] Le Ray C, Carayol M, Zeitlin J, Breart G, Goffinet F, Group PS, et al. Level of perinatal care of the maternity unit and rate of cesarean in low-risk nulliparas. Obstet Gynecol 2006;107:1269-77.

[31] Kramer MS, Seguin L, Lydon J, Goulet L. Socio-economic disparities in pregnancy outcome: why do the poor fare so poorly? Paediatr Perinat Epidemiol 2000; $14: 194-210$

[32] Fairley L. Social class inequalities in perinatal outcomes: Scotland 1980-2000. J Epidemiol Community Health 2006;60:31-6. 